\title{
Consistency-driven methodology to manage incomplete linguistic preference relation: A perspective based on personalized individual semantics
}

\author{
Cong-Cong Li, Yucheng Dong, Francisco Chiclana, Enrique Herrera-Viedma
}

\begin{abstract}
In linguistic decision making problems there may be cased when decision makers will not be able to provide complete linguistic preference relations. However, when estimating unknown linguistic preference values in incomplete preference relations, the existing research approaches ignore the fact that words mean different things for different people, i.e. decision makers have personalized individual semantics (PISs) regarding words. To manage incomplete linguistic preference relations with PISs, in this paper we propose a consistency-driven methodology both to estimate the incomplete linguistic preference values and to obtain the personalized numerical meanings of linguistic values of the different decision makers. The proposed incomplete linguistic preference estimation method combines the characteristic of the personalized representation of decision makers and guarantees the optimum consistency of incomplete linguistic preference relations in the implementation process. Numerical examples and a comparative analysis are included to justify the feasibility of the PISs based incomplete linguistic preference estimation method.
\end{abstract}

Index Terms_Personalized individual semantics, incomplete linguistic preference relation, consistency, linguistic decision making

This work was supported by the grant (Nos. 71901182 and 71871149) from NSF of China and the grants (Nos. sksyl201705 and 2018hhs-58) from Sichuan University, the grant (No. YJSY-DSTD201918) from Southwest Jiaotong Universtiy, and supported by Spanish State Research Agency through the project PID2019-103880RB-I00/AEI/10.13039/501100011033.

(Corresponding author: Yucheng Dong)

C.C. $\mathrm{Li}$ is with the School of Economics and Management, Southwest Jiaotong University, Chengdu 610031, China (e-mail: congcongli@swjtu.edu.cn).

Y. Dong is with Business School, Sichuan University, Chengdu 610065, China (e-mail: ycdong@scu.edu.cn).

F. Chiclana is with the Institute of Artificial Intelligence, School of Computer Science and Informatics, De Montfort University, Leicester LE1 9BH, U.K., and also with the Andalusian Research Institute in Data Science and Computational Intelligence, University of Granada, 18071 Granada, Spain (e-mail: chiclana@dmu.ac.uk).

E. Herrera-Viedma is with the Andalusian Research Institute in Data Science and Computational Intelligence, Dept. of Computer Science and AI, University of Granada, Granada 18071, Spain, and the Department of Electrical and Computer Engineering, Faculty of Engineering, King Abdulaziz University, Jeddah 21589, Saudi Arabia (e-mail: viedma@ decsai.ugr.es).

\section{INTRODUCTION}

In real group decision making (GDM) activity, decision makers may prefer to use linguistic information instead of numerical numbers to represent their preferences. In this case, we deal with what is called a linguistic group decision making (LGDM). The LGDM problem aims to finding the best alternative(s) from a set of potential alternatives based on the linguistic preferences expressed by a group of decision makers [2], [11]. Particularly, linguistic preference relations [13], [40] are commonly used in LGDM to express decision makers' preferences over alternatives.

A difficulty in dealing with preference relations in GDM problems is the missing of some of the expected preference information values [10], [15], [22], [32]. Experts may not provide all the expected preference degree between two or more alternatives because the number of alternatives is high or because lack of knowledge on some of the pairwise comparisons. In such situation, experts provide incomplete preference relations. Generally, two types of methods have been proposed to manage incomplete preference relations based on consistency measurements:

(i) The iterative procedure, which estimates missing values between two alternatives based on all possible indirect paths between such alternatives, using intermediate alternatives, for which preference values are known.

(ii) The optimization-based procedure that obtains a complete linguistic preference relation with optimum consistency.

The iterative procedure and optimization-based procedure are both extensively investigated in various types of preference relations, such as additive preference relation (or fuzzy preference relation) [9], [27], [51], multiplicative preference relation [5], [28], interval-valued preference relation [34], [35], [44]. These approaches are also reported to manage incomplete linguistic preference relations [1], [3], [45] through linguistic additive consistency measurement [4], [7], [41], [42]. Based on the concept of additive consistency, Alonso et al. [1] proposed the iterative procedure to obtain the complete linguistic preference relations, which only uses the available values provided by the experts. Cabrerizo et al. [3] developed the iterative procedure for LGDM under an incomplete unbalanced linguistic environment. Zhao et al. [45] proposed the optimization-based model to estimates the missing values in 
incomplete linguistic preference relations based on consistency measurement. The state-of-the-art survey about the management of incomplete preference relations has been presented in [21], [32].

It is argued and accepted that words mean different things for different people [25], [26] and, therefore, in LGDM decision makers have personalized individual semantics (PISs) [18] regarding words. For example, if two researchers reviewing an article think that the reviewed article is interesting, it may be the case that the word "interesting" would have different numerical interpretations or meanings to them. If the two researchers are asked to do semantics modelling of the word "interesting", the numerical meaning of word "interesting" may be 0.9 for one researcher and 0.7 for the other researcher. The difference in the numerical meanings shows the PISs for researchers. It is even recognized that the concept of type-1 fuzzy set is not sufficient to represent the multiple meanings of words and, therefore, the concept of type-2 fuzzy set was proposed [25]. However, the type-2 fuzzy set concept does not capture in its definition the specific decision makers' semantics. It is noted that to represent the PISs, the consistency-driven optimization model to personalize individual semantics of words for decision makers has been initiated in [18]. Based on the PIS model in [18], Huang et al. [17] proposed a new consensus reaching process in LGDM. Li et al. [19], [20], Zhang et al. [48] and Tang et al. [30], [31] studied the consistency-driven approaches in hesitant LGDM, large-scale LGDM, and distribution linguistic GDM, respectively, to show the PISs. The PIS model has also been applied in failure modes and effects analysis [49] and opinion dynamics [23].

An interesting, and worth to investigate, issue is that of estimating the missing values of the incomplete linguistic preference relations by considering the different individual semantics of decision makers. However, although the existing studies provide various methods to manage incomplete linguistic preference relations, they do not consider the decision makers' PISs. Therefore, in this paper we propose a two-phase consistency-driven methodology to manage incomplete linguistic preference relations with PISs:

(1) In the first phase, a PIS based consistency-driven optimization (PIS-CDO) model is proposed to find out the set of possible personalized numerical scales (PNSs) that guarantee the optimum consistency of incomplete preference relations, which constitutes a foundational constraint condition to manage incomplete linguistic preference relations.

(2) In the second phase, the incomplete preference estimation based consistency-driven optimization (IPE-CDO) model to estimate the missing values in incomplete linguistic preference relations with PISs is developed.

Finally, we further illustrate the use and explain the feasibility of the incomplete preference estimation method with PISs. Numerical examples for the proposed method are provided and a comparative study with the existing methods, which does not implement PISs, is carried out. The main features of the incomplete preference estimation method with PISs are that it integrates the characteristic of the personalized representation of linguistic preferences, and guarantees the optimum consistency of incomplete linguistic preference relations.

The rest of this paper is arranged as follows. In Section II, we present some basic preliminaries regarding LGDM that is necessary to develop our proposal. Then, in Section III the PIS-CDO model and the IPE-CDO model are both proposed to manage incomplete linguistic preference relations with PISs. Next, Section IV provides numerical examples to illustrate our proposal while Section V includes details of a comparative study with the existing studies that do not implement PISs by numerical analysis. Finally, Section VI concludes this paper.

\section{PRELIMINARIES}

In this section, we introduce the basic knowledge regarding the 2-tuple linguistic model, linguistic preference relations, and the numerical scale model based on PIS, which is necessary to develop our proposal.

\section{A. The 2-tuple linguistic model}

Let $S=\left\{s_{i} \mid i=0,1, \ldots, g\right\}$ be a linguistic term set. The linguistic term $s_{i}$ represents a possible value of a linguistic variable [24], [46]. Herrera and Martínez [14] proposed the below 2-tuple linguistic model for computing with words.

Definition 1 [14]. Let $S$ be defined as above, let $\beta \in[0, g]$ be a value representing the result of a symbolic aggregation operation. The 2-tuple linguistic model defines the transformation functions between 2-tuples and numerical values:

$$
\Delta:[0, g] \rightarrow \bar{S}
$$

being

$$
\Delta(\beta)=\left(s_{i}, \alpha\right) \text {, }
$$$$
\text { with }\left\{\begin{array}{lr}
s_{i}, & i=\operatorname{round}(\beta) \\
\alpha=\beta-i, \alpha \in[-0.5,0.5)
\end{array}\right.
$$

The inverse function of $\Delta, \Delta^{-1}: \bar{S} \rightarrow[0, g]$ is defined as $\Delta^{-1}\left(s_{i}, \alpha\right)=i+\alpha$.

The computational model for linguistic 2-tuples [14] is the following:

(1) Comparison operator for linguistic 2-tuples: Let $\left(s_{k}, \alpha\right)$ and $\left(s_{l}, \gamma\right)$ be any two 2-tuples, then

(i) if $k>l$, then $\left(s_{k}, \alpha\right)$ is larger than $\left(s_{l}, \gamma\right)$.

(ii) if $k=l$, then

(a) if $\alpha=\gamma$, then $\left(s_{k}, \alpha\right),\left(s_{l}, \gamma\right)$ represents the same information.

(b) if $\alpha>\gamma$, then $\left(s_{k}, \alpha\right)$ is larger than $\left(s_{l}, \gamma\right)$.

(2) Negation operator for linguistic 2-tuples:

$$
\operatorname{Neg}\left(\left(s_{i}, \alpha\right)\right)=\Delta\left(g-\left(\Delta^{-1}\left(s_{i}, \alpha\right)\right)\right)
$$

(3) The aggregation operators for linguistic 2-tuples were defined in [14], and their details are omitted herein.

\section{B. Linguistic preference relations and consistency measurements}

Let $A=\left\{A_{1}, A_{2}, \ldots, A_{n}\right\}$ be a set of alternatives. When decision makers pairwise compare alternatives $\left(A_{i}, A_{j}\right)$ using the linguistic term set $S$, they construct a linguistic preference 
relation $L=\left(l_{i j}\right)_{n \times n}$, where $l_{i j}$ denotes the linguistic preference degree of $A_{i}$ over $A_{j}$.

Definition 2 [12], [13]. The linguistic preference relation $L=\left(l_{i j}\right)_{n \times n}$ is complete if $l_{i j} \in S$ and $l_{i j}=\operatorname{Neg}\left(l_{j i}\right)$ for all $i, j=1,2, \ldots, n$.

Additive transitivity [1], [8], [15] is often used in decision making to measure the consistency of preference relations. The consistency measurement of linguistic preference relations based on the 2-tuple linguistic model is provided as below.

Definition 3 [1]. Let $L=\left(l_{i j}\right)_{n \times n}$ be a complete linguistic preference relation based on $S$. The consistency index of $L$ is defined as follows,

$$
\begin{aligned}
& C I(L)=1-\frac{2}{3 g n(n-1)(n-2)} \sum_{i, j, k=1}^{n} \mid \Delta^{-1}\left(l_{i j}\right)+\Delta^{-1}\left(l_{j k}\right)- \\
& \Delta^{-1}\left(l_{i k}\right)-g / 2 \mid
\end{aligned}
$$

Clearly, $C I(L) \in[0,1]$. A larger value of $C I(L)$ indicates a better consistency of $L$.

In some cases decision makers cannot provide linguistic preferences for all the possible pairs of alternatives, which leads to the practical use of incomplete linguistic preference relations in decision making, a concept that is provided below.

Definition 4 [21]. $L=\left(l_{i j}\right)_{n \times n}$ is an incomplete linguistic preference relation when some of its elements are missing or unknown (not provided by the decision maker and denoted by the symbol " $x$ " herein), while the known elements (provided by the decision maker) satisfy $l_{i j} \in S$ and $l_{i j}=\operatorname{Neg}\left(l_{j i}\right)$.

\section{The PIS-based numerical scale model}

Dong et al. [6] defined the below concept of the numerical scale as an extension of the linguistic 2-tuples.

Definition 5 [6]. Let $S=\left\{s_{0}, s_{1}, \ldots, s_{g}\right\}$ be a linguistic term set, and $R$ be the set of real numbers. A function $N S: S \rightarrow R$ is called a numerical scale of $S$, and $N S\left(s_{i}\right)$ is the numerical index of $s_{i}$.

Definition 6 [6]. Let $S$ be defined as above. The numerical scale $N S$ for $\left(s_{i}, \alpha\right)$ is defined as follows:

$N S\left(s_{i}, \alpha\right)=\left\{\begin{array}{l}N S\left(s_{i}\right)+\alpha \times\left(N S\left(s_{i+1}\right)-N S\left(s_{i}\right)\right), \alpha \geq 0 \\ N S\left(s_{i}\right)+\alpha \times\left(N S\left(s_{i}\right)-N S\left(s_{i-1}\right)\right), \alpha<0\end{array}\right.$

If $N S\left(s_{i}\right)<N S\left(s_{i+1}\right)$, for $i=0,1, \ldots, g-1$, then the $N S$ on $S$ is ordered.

The inverse operator of the numerical scale $N S$ of Definition 6 is [6]

$$
N S^{-1}: R \rightarrow \bar{S}
$$

with

$$
\begin{aligned}
& N S^{-1}(r) \\
& =\left\{\begin{array}{c}
\left(s_{i}, \frac{r-N S\left(s_{i}\right)}{N S^{k}\left(s_{i+1}\right)-N S^{k}\left(s_{i}\right)}\right), \quad N S\left(s_{i}\right)<r<\frac{N S\left(s_{i}\right)+N S\left(s_{i+1}\right)}{2} \\
\left(s_{i}, \frac{r-N S\left(s_{i}\right)}{N S\left(s_{i}\right)-N S\left(s_{i-1}\right)}\right), \quad \frac{N S\left(s_{i-1}\right)+N S\left(s_{i}\right)}{2} \leq r \leq N S\left(s_{i}\right)
\end{array}\right.
\end{aligned}
$$

The desired property of the above numerical scale to connect the 2-tuple linguistic models was discussed in [7].

The consistency measurement of complete linguistic preference relation based on $N S$ is provided below.

Definition 7 [6], [15]. $L=\left(l_{i j}\right)_{n \times n}$ is a consistent complete linguistic preference relation with respect to the ordered numerical scale $N S$ if for all $i, j, k=1,2, \ldots, n: N S\left(l_{i j}\right) \in[0,1]$ and $N S\left(l_{i j}\right)+N S\left(l_{j k}\right)-N S\left(l_{i k}\right)=0.5$.

Definition 8 [6]. Let $L=\left(l_{i j}\right)_{n \times n}$ be a complete linguistic preference relation and $N S$ be the numerical scale on $S$. Then, the consistency index of $L$ with respect to $N S$ is

$C I(L)=1-\frac{4}{n(n-1)(n-2)} \sum_{i<j<k}^{n}\left|N S\left(l_{i j}\right)+N S\left(l_{j k}\right)-N S\left(l_{i k}\right)-0.5\right|(6)$ with $N S\left(l_{i j}\right) \in[0,1]$ for all $i, j=1,2, \ldots, n$.

To handle the fact that words have multiple meanings for different people, Li et al. [18] presented a framework to handle linguistic information in the LGDM with PISs (Fig.1), which includes three processes: the individual semantics translation, numerical computation and individual semantics retranslations.

The individual semantics translation is used to translate the linguistic terms into the PISs defined by the numerical values, in which the output activated the numerical computation to obtain a numerical value. The individual semantics retranslation is used to retranslate the output of numerical scales into linguistic values.

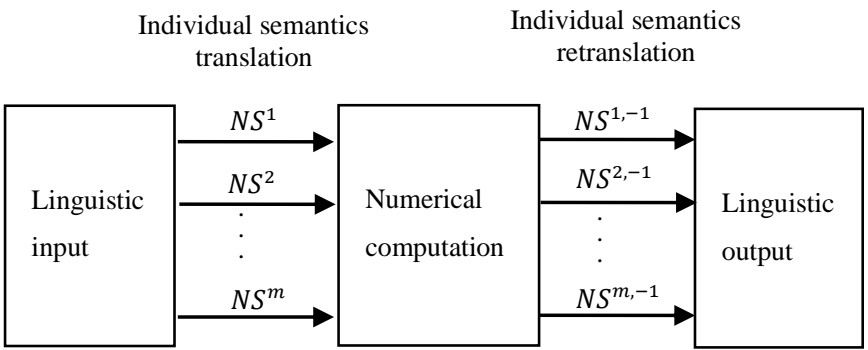

Fig.1. The framework for the linguistic model with PISs

In Fig. $1, N S^{k}$ is the ordered numerical scale on $S$ of decision maker $e_{k}(k=1,2, \ldots, m)$, and the value $N S^{k}\left(s_{i}\right)$ represents the individual semantics of such decision maker $e_{k}$ on the term $s_{i}(i=0,1, \ldots, g)$. Furthermore, Li et al. [18-20] proposed consistency-driven optimization models to obtain the PNSs of linguistic terms for decision makers under different linguistic decision making contexts.

\section{CONSISTENCY-BASED PIS METHOD TO MANAGE INCOMPLETE LINGUISTIC PREFERENCE RELATIONS}

In LGDM, the PISs reflect the different understanding of words among decision makers. To manage incomplete linguistic preference relations in a PIS context, in this section we propose a consistency-based methodology to estimate the unknown values of incomplete linguistic preference relations and to obtain the PNSs of linguistic expressions for decision makers.

\section{A. The framework for the incomplete preference estimation method with PISs}

Similar to the consistency measurement of a complete linguistic preference relation (see Definitions 3 and 8), we define the measure of consistency of an incomplete linguistic preference relation based on the additive transitivity. The condition that guarantees that the consistency of an incomplete linguistic preference relation $L=\left(l_{i j}\right)_{n \times n}$ can be measured is 
the existence of at least three different preference values, $l_{i j}, l_{j k}, l_{i k}(i \neq j \neq k)$, that are known.

When studying incomplete linguistic preference relations, the following sets are needed,

$$
\begin{gathered}
V=\left\{(i, j) \mid l_{i j} \text { is known },(i, j) \in\{1,2, \ldots, n\}, i \neq j\right\} \\
A T V=\{(i, j, k) \mid(i, j),(j, k),(i, k) \in V\}
\end{gathered}
$$

$V$ is the set of pairs of alternatives for which the decision makers provide linguistic preference values; $A T V$ is the set of triplets of alternatives $A_{i}, A_{j}, A_{k}$ for which the preference degrees over these alternatives are known. The consistency measurement for incomplete linguistic preference relations is provided as follows,

Definition 9. Let $L=\left(l_{i j}\right)_{n \times n}$ be an incomplete linguistic preference relation. Then the consistency index of $L$ is

$C I(L)=1-\frac{2}{3 * \# A T V} \sum_{i<j<k ;(i, j, k) \in A T V}^{n} \mid N S\left(l_{i j}\right)+N S\left(l_{j k}\right)-$ $N S\left(l_{i k}\right)-0.5$

with $N S\left(l_{i j}\right) \in[0,1]$ for all $i, j=1,2, \ldots, n$.

As mentioned before, being consistent implies that the decision makers are neither random nor illogical when expressing preferences. In order to estimate the missing values and also to guarantee the consistency of an incomplete linguistic preference relation, we propose a two-phase consistency-driven methodology:

(1) In the first phase, a PIS-CDO model is developed to optimize the consistency of the incomplete linguistic preference relation by generating the set of all possible PNSs for the known values.

(2) In the second phase, an IPE-CDO model to estimate the missing values of incomplete linguistic preference relation with PISs is established. This model guarantees the optimum consistency of both the original incomplete linguistic preference relation and its complete linguistic preference relation derived from the estimation process.

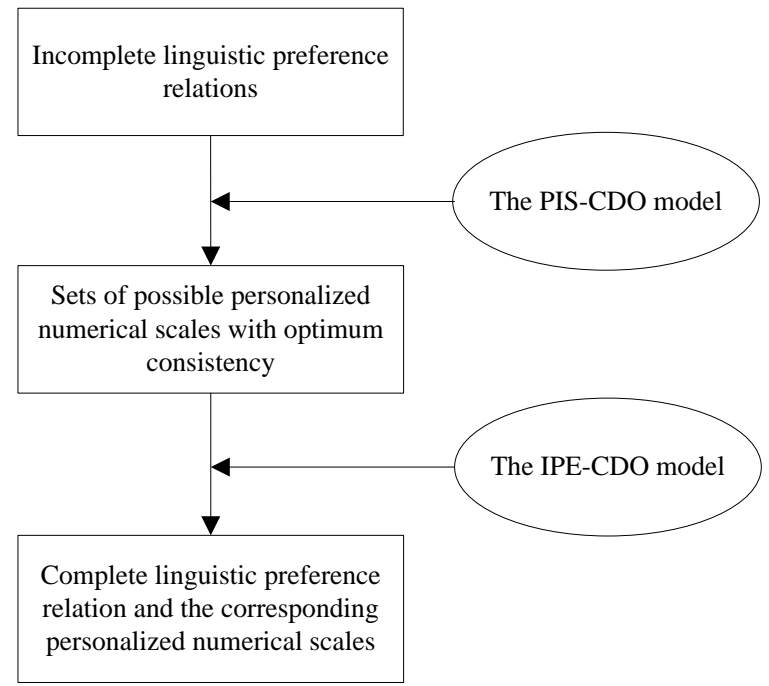

Fig.2. The framework for managing incomplete linguistic preference relations with PISs

Fig. 2 depicts the framework of the proposed methodology to manage incomplete linguistic preference relations.

\section{B. The PIS-CDO model with incomplete linguistic preference} relations

This subsection provides a PIS-CDO model to obtain the set of all possible PNSs for an incomplete linguistic preference relation that guarantees it has the maximum consistency.

Let $L^{k}$ be the incomplete linguistic preference relation provided by decision maker $e_{k}$. Let $N S^{k}$ be the numerical scale associated with $e_{k}$. As per the previous definitions, let $V^{k}$ and $A T V^{k}$ be the sets of pairs and triplets of alternatives with known linguistic values given by decision maker $e_{k}$ :

$$
\begin{gathered}
V^{k}=\left\{(i, j) \mid l_{i j}^{k} \text { is known, }(i, j) \in\{1,2, \ldots, n\}, i \neq j\right\} \\
A^{k} V^{k}=\left\{(i, j, z) \mid(i, j),(j, z),(i, z) \in V^{k}\right\}
\end{gathered}
$$

As we aim to maximize the consistency of incomplete linguistic preference relations, the objective function of the PIS-CDO model will be:

$\max C I\left(L^{k}\right)$

where $C I\left(L^{k}\right)$ is defined as per expression (7) of Definition 9.

The range of the numerical scale for each linguistic term associated with decision maker $e_{k}$ is

$N S^{k}\left(s_{i}\right)\left\{\begin{array}{l}=0, \quad i=0 \\ \in\left[\frac{i-0.5}{g}, \frac{i+0.5}{g}\right), \quad i=1,2, \ldots, g-1 ; i \neq \frac{g}{2} \\ =0.5, \quad i=\frac{g}{2} \\ =1, \quad i=g\end{array}\right.$

Thus, the constructed PIS-CDO model with incomplete linguistic preference relation $L^{k}$ is

$$
\left\{\begin{array}{l}
\max C I\left(L^{k}\right)=1-\frac{2}{3 * \# A T V^{k}} \\
\sum_{i<j<z ;(i, j, z) \in A T V^{k}}^{n}\left|N S^{k}\left(l_{i j}^{k}\right)+N S^{k}\left(l_{j z}^{k}\right)-N S^{k}\left(l_{i z}^{k}\right)-0.5\right| \\
\text { s.t. } S^{k}\left(s_{0}\right)=0 \\
N S^{k}\left(s_{q}\right) \in\left(\frac{q-0.5}{g}, \frac{q+0.5}{g}\right], \quad q=1,2, \ldots, g-1 ; q \neq g / 2 \\
N S^{k}\left(s_{\frac{g}{2}}\right)=0.5 \\
N S^{k}\left(s_{g}\right)=1
\end{array}\right.
$$

By solving Model (10), we generate the set of possible PNSs of linguistic terms for $e_{k}$, denoted $A P S=$ $\left\{\left(N S^{k}\left(s_{0}\right), N S^{k}\left(s_{1}\right), \ldots, N S^{k}\left(s_{g}\right)\right) ; \ldots\right\}$, that guarantee the maximum consistency of the provided incomplete linguistic preference relation $L^{k}$. The obtained optimum consistency index of $L^{k}$ is denoted $\operatorname{OCI}\left(L^{k}\right)$ in this paper.

Model (10) provides a novel approach to measure the consistency of incomplete linguistic preference relations by setting the PNSs for linguistic terms. The obtained results will be used in the IPE-CDO model to make sure the optimum consistency of the incomplete linguistic preference relation will not be destroyed.

\section{The IPE-CDO model to estimate the missing values}


This subsection proposes an IPE-CDO model to estimate the missing values of an incomplete linguistic preference relation and its personalized numerical meanings for the corresponding decision maker, while guaranteeing that the obtained complete linguistic preference relation has maximum consistency level.

Let $L^{k}$ be defined as before. Let $\bar{L}^{k}=\left(\bar{l}_{i j}^{k}\right)_{n \times n}$ be the complete linguistic preference relation associated to $L^{k}$. The objective of the optimization model with PISs that estimates the missing values will be

$\max C I\left(\bar{L}^{k}\right)$

where $\operatorname{CI}\left(\bar{L}^{k}\right)$ is defined as per expression (6) of Definition 8.

Let $\operatorname{OCI}\left(L^{k}\right)$ be the consistency index of the incomplete linguistic preference relation $L^{k}$ obtained from Model (10). To guarantee the optimum consistency of $L^{k}$ is not destroyed in the process of estimating missing values, the PNSs of the known linguistic values provided by decision maker $e_{k}$ should belong to the set $A P S$, and therefore

$$
\left\{N S^{k}\left(\bar{l}_{i j}^{k}\right) \mid \vec{l}_{i j}^{k} \in V^{k}\right\} \subseteq A P S
$$

Eq. (12) can also be expressed as follows:

$$
\begin{aligned}
& 1-\frac{2}{3 \times A T V^{k}} \sum_{i<j<z ;(i, j, z) \in A T V^{k}}^{n} \mid N S^{k}\left(\bar{l}_{i j}^{k}\right)+N S^{k}\left(\bar{l}_{j z}^{k}\right) \\
& -N S^{k}\left(\bar{l}_{i z}^{k}\right)-0.5 \mid=O C I\left(L^{k}\right)
\end{aligned}
$$

where $\# A T V^{k}$ is the cardinality of set $A T V^{k}$.

The range for the numerical scales of linguistic terms for decision maker $e_{k}$ is set as in Model (10), i.e.,

$$
N S^{k}\left(s_{q}\right)\left\{\begin{array}{l}
=0, \quad q=0 \\
\in\left[\frac{q-0.5}{g}, \frac{q+0.5}{g}\right), \quad q=1,2, \ldots, g-1 ; i \neq \frac{g}{2} \\
=0.5, \quad q=\frac{g}{2} \\
=1, \quad q=g
\end{array}\right.
$$

In constructing the complete linguistic preference relation $\bar{L}^{k}$, its elements need to fulfill the following constraints

$$
\begin{cases}l_{i j}^{k}=l_{i j}^{k}, & l_{i j}^{k} \neq x \\ l_{i j}^{k} \in S, & l_{i j}^{k}=x\end{cases}
$$

Bringing all the above together results in the following IPE-CDO model for estimating the missing values of $L^{k}$

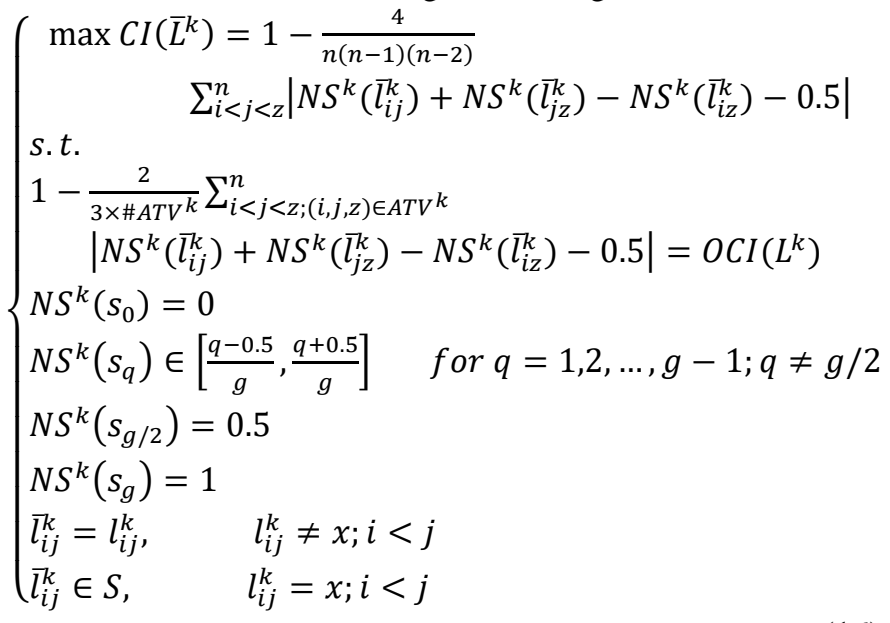

By solving Model (16), we estimate the missing values of $L^{k}$ to generate its complete linguistic preference relation $\bar{L}^{k}$ and further obtain the PNSs of linguistic terms for $e_{k}$, $N S^{k}\left(s_{0}\right), N S^{k}\left(s_{1}\right), \ldots, N S^{k}\left(s_{g}\right)$. Besides, we also obtain the optimum consistency index of $\bar{L}^{k}, O C I\left(\bar{L}^{k}\right)$. The obtained PNSs may be different for different decision makers, which agree with the actual different understanding of words by different decision makers.

From this two-phase method for managing incomplete linguistic preference relations, it is clear that the proposed methodology provides a novel consistency index of an incomplete linguistic preference relations by considering the decision makers' PISs in estimating the missing values when constructing its complete linguistic preference relation.

\section{NUMERICAL EXAMPLE}

In this section, we present numerical examples to illustrate the proposed consistency-driven optimization models in managing incomplete linguistic preference relations.

Let $S$ be the following linguistic term set

$$
\begin{gathered}
S=\left\{s_{0}=\text { extremely poor }, s_{1}=\text { very poor }, s_{2}=\text { poor }, s_{3}\right. \\
=\text { fair }, s_{4}=\text { good, } s_{5}=\text { very good, } s_{6} \\
=\text { extremely good }\}
\end{gathered}
$$

Let $E=\left\{e_{1}, e_{2}, e_{3}, e_{4}, e_{5}, e_{6}, e_{7}, e_{8}\right\} \quad$ and $A=\left\{A_{1}, A_{2}\right.$, $\left.A_{3}, A_{4}, A_{5}\right\}$ be the set of decision makers and alternatives, respectively. We assume the following incomplete linguistic preference relations are provided by $e_{k}(k=1,2, \ldots, 8)$,

$$
\begin{aligned}
& L^{1}=\left(\begin{array}{ccccc}
- & s_{4} & s_{1} & x & x \\
s_{2} & - & s_{2} & s_{3} & x \\
s_{5} & s_{4} & - & s_{0} & x \\
x & s_{3} & s_{6} & - & s_{2} \\
x & x & x & s_{4} & -
\end{array}\right) \\
& L^{2}=\left(\begin{array}{ccccc}
- & s_{6} & x & x & s_{0} \\
s_{0} & - & x & s_{3} & s_{4} \\
x & x & - & x & x \\
x & s_{3} & x & - & s_{6} \\
s_{6} & s_{2} & x & s_{0} & -
\end{array}\right) \\
& L^{3}=\left(\begin{array}{ccccc}
- & x & x & x & x \\
x & - & s_{1} & s_{5} & s_{0} \\
x & s_{5} & - & s_{6} & s_{2} \\
x & s_{1} & s_{0} & - & x \\
x & s_{6} & s_{4} & x & -
\end{array}\right) \\
& L^{4}=\left(\begin{array}{ccccc}
- & s_{1} & x & s_{4} & s_{0} \\
s_{5} & - & x & s_{5} & x \\
x & x & - & x & x \\
s_{2} & s_{1} & x & - & s_{6} \\
s_{6} & x & x & s_{0} & -
\end{array}\right) \\
& L^{5}=\left(\begin{array}{ccccc}
- & x & s_{2} & s_{1} & x \\
x & - & s_{5} & s_{0} & x \\
s_{4} & s_{1} & - & s_{4} & x \\
s_{5} & s_{6} & s_{2} & - & x \\
x & x & x & x & -
\end{array}\right) \\
& L^{6}=\left(\begin{array}{ccccc}
- & s_{5} & s_{1} & x & s_{0} \\
s_{1} & - & s_{6} & s_{2} & s_{4} \\
s_{5} & s_{0} & - & s_{3} & x \\
x & s_{4} & s_{3} & - & x \\
s_{6} & s_{2} & x & x & -
\end{array}\right)
\end{aligned}
$$




$$
\begin{aligned}
L^{7} & =\left(\begin{array}{ccccc}
- & x & x & x & s_{1} \\
x & - & s_{6} & s_{5} & s_{4} \\
x & s_{0} & - & x & s_{5} \\
x & s_{1} & x & - & s_{2} \\
s_{5} & s_{2} & s_{1} & s_{4} & - \\
- & x & s_{5} & x & s_{1} \\
x & - & x & s_{4} & s_{5} \\
s_{1} & x & - & x & s_{2} \\
x & s_{2} & x & - & s_{3} \\
s_{5} & s_{1} & s_{4} & s_{3} & -
\end{array}\right) \\
L^{8} & =\left(\begin{array}{cccc} 
&
\end{array}\right.
\end{aligned}
$$

(1) Phase I: The PIS-CDO model with incomplete linguistic preference relations.

In this phase optimum consistency of $L^{k}=\left(l_{i j}^{k}\right)_{5 \times 5}(k=$ $1,2, \ldots, 8)$ is obtained via the generation of the PNSs for the linguistic terms. As an example, we illustrate this phase with $L^{1}$ by building the consistency-driven optimization model (10) with following Eqs. (17)-(24).

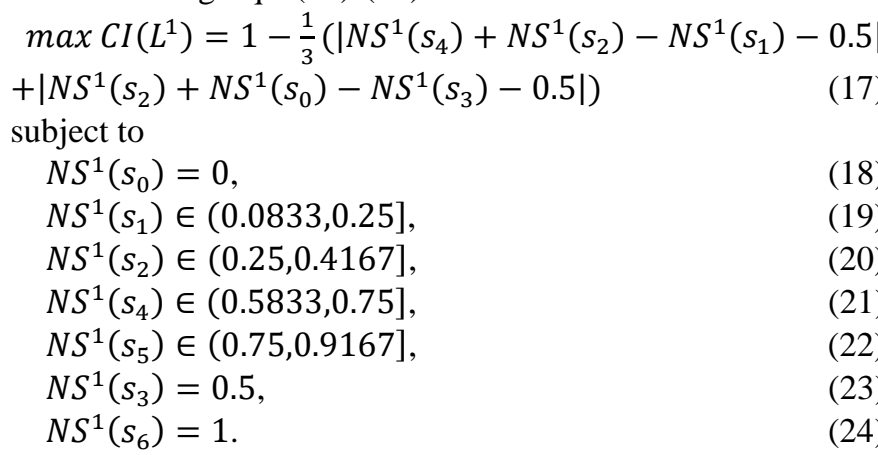

The solution of this model results in $\operatorname{OCI}\left(L^{1}\right)=0.722$ with the set of PNSs, APS, that can include several possible PNSs, such as

$$
\begin{gathered}
\left\{N S^{1}\left(s_{0}\right)=0 ; N S^{1}\left(s_{1}\right)=0.25 ; N S^{1}\left(s_{2}\right)=\right. \\
0.3331 ; N S^{1}\left(s_{3}\right)=0.5 ; N S^{1}\left(s_{4}\right)=0.5833 ; N S^{1}\left(s_{5}\right) \in \\
\left.(0.75,0.9167], N S^{1}\left(s_{6}\right)=1\right\} \subseteq A P S,
\end{gathered}
$$

and

$$
\left\{N S^{1}\left(s_{0}\right)=0, N S^{1}\left(s_{1}\right)=0.249, N S^{1}\left(s_{2}\right)=0.375,\right.
$$$$
N S^{1}\left(s_{3}\right)=0.5, N S^{1}\left(s_{4}\right)=0.583, N S^{1}\left(s_{5}\right) \in[0.75,0.9167] \text {, }
$$$$
\left.N S^{1}\left(s_{6}\right)=1\right\} \subseteq A P S \text {. }
$$

Similarly, we obtain the following optimum consistency indexes for $L^{k}(k=2,3, \ldots, 8): \quad \operatorname{OCI}\left(L^{2}\right)=0.5$, $\operatorname{OCI}\left(L^{3}\right)=0.995, \quad \operatorname{OCI}\left(L^{4}\right)=0.6386, \quad \operatorname{OCI}\left(L^{5}\right)=0.687$, $\operatorname{OCI}\left(L^{6}\right)=0.426, \operatorname{OCI}\left(L^{7}\right)=0.829$ and $\operatorname{OCI}\left(L^{8}\right)=0.91$.

The obtained optimum consistency indexes $\operatorname{OCI}\left(L^{k}\right)(k=$ $1,2, \ldots, 8)$ will be used as a constraint condition in Phase II to further guarantee the PISs of decision makers in solving the complete linguistic preference relation $\bar{L}^{k}$.

(2) Phase II: The IPE-CDO model to estimate the missing values.

In this phase, solving Model (16) results in the complete linguistic preference relation $\bar{L}^{k}$, i.e., the missing values of $L^{k}(k=1,2, \ldots, 8)$ are estimated, and in the personalized numerical meanings for decision makers $e_{k}$, while ensuring the consistency of $\bar{L}^{k}$ at the same time. Below, we illustrate this with the incomplete linguistic preference relation $L^{1}$ (See Eqs. (25)-(36)). $\max C I\left(\bar{L}^{1}\right)=1-\frac{1}{15} \sum_{i<j<z}^{5}\left|N S^{1}\left(\bar{l}_{i j}^{1}\right)+N S^{1}\left(\bar{l}_{j z}^{1}\right)-N S^{1}\left(\bar{l}_{i z}^{1}\right)-0.5\right|$

subject to

$$
\begin{aligned}
& 1-\frac{1}{3}\left(\left|N S^{1}\left(\bar{l}_{12}^{1}\right)+N S^{1}\left(\bar{l}_{23}^{1}\right)-N S^{1}\left(\bar{l}_{13}^{1}\right)-0.5\right|+\right. \\
& \left.\left|N S^{1}\left(\bar{l}_{23}^{1}\right)+N S^{1}\left(\bar{l}_{34}^{1}\right)-N S^{1}\left(\bar{l}_{24}^{1}\right)-0.5\right|\right)=0.722, \\
& N S^{1}\left(s_{0}\right)=0, \\
& N S^{1}\left(s_{1}\right) \in(0.0833,0.25], \\
& N S^{1}\left(s_{2}\right) \in(0.25,0.4167], \\
& N S^{1}\left(s_{4}\right) \in(0.5833,0.75], \\
& N S^{1}\left(s_{5}\right) \in(0.75,0.9167], \\
& N S^{1}\left(s_{3}\right)=0.5, \\
& N S^{1}\left(s_{6}\right)=1, \\
& \bar{l}_{12}^{1}=s_{4} ; \bar{l}_{13}^{1}=s_{1} ; \bar{l}_{23}^{1}=s_{2}, \\
& \bar{l}_{24}^{1}=s_{3} ; \bar{l}_{34}^{1}=s_{0} ; \bar{l}_{45}^{1}=s_{2}, \\
& \bar{l}_{14}^{1} \in S ; \bar{l}_{15}^{1} \in S ; \bar{l}_{25}^{1} \in S ; \bar{l}_{35}^{1} \in S .
\end{aligned}
$$

The solution of this model yields the following missing value estimations: $\bar{l}_{14}^{1}=s_{2}, \bar{l}_{15}^{1}=s_{1}, \bar{l}_{25}^{1}=s_{1}, \bar{l}_{35}^{1}=s_{2}$. Thus, the complete linguistic preference relation $\bar{L}^{1}$ is

$$
\bar{L}^{1}=\left(\begin{array}{ccccc}
- & s_{4} & s_{1} & s_{2} & s_{1} \\
s_{2} & - & s_{2} & s_{3} & s_{1} \\
s_{5} & s_{4} & - & s_{0} & s_{2} \\
s_{4} & s_{3} & s_{6} & - & s_{2} \\
s_{5} & s_{5} & s_{4} & s_{4} & -
\end{array}\right)
$$

The PNSs for decision maker $e_{1}$ are: $N S^{1}\left(s_{0}\right)=0$, $N S^{1}\left(s_{1}\right)=0.249, N S^{1}\left(s_{2}\right)=0.375, N S^{1}\left(s_{3}\right)=0.5$, $N S^{1}\left(s_{4}\right)=0.584$, and $N S^{1}\left(s_{6}\right)=1$.

Similarly, we obtain the rest of complete linguistic preference relations

$$
\begin{aligned}
& \bar{L}^{2}=\left(\begin{array}{ccccc}
- & s_{6} & s_{1} & s_{0} & s_{0} \\
s_{0} & - & s_{3} & s_{3} & s_{4} \\
s_{5} & s_{3} & - & s_{1} & s_{4} \\
s_{6} & s_{3} & s_{5} & - & s_{6} \\
s_{6} & s_{2} & s_{2} & s_{0} & -
\end{array}\right) \\
& \bar{L}^{3}=\left(\begin{array}{ccccc}
- & s_{3} & s_{1} & s_{4} & s_{0} \\
s_{3} & - & s_{1} & s_{5} & s_{0} \\
s_{5} & s_{5} & - & s_{6} & s_{2} \\
s_{2} & s_{1} & s_{0} & - & s_{0} \\
s_{6} & s_{6} & s_{4} & s_{6} & -
\end{array}\right) \\
& \bar{L}^{4}=\left(\begin{array}{ccccc}
- & s_{1} & s_{1} & s_{4} & s_{0} \\
s_{5} & - & s_{3} & s_{5} & s_{1} \\
s_{5} & s_{3} & - & s_{5} & s_{1} \\
s_{2} & s_{1} & s_{1} & - & s_{6} \\
s_{6} & s_{5} & s_{5} & s_{0} & -
\end{array}\right) \\
& \bar{L}^{5}=\left(\begin{array}{ccccc}
- & s_{3} & s_{2} & s_{1} & s_{0} \\
s_{3} & - & s_{5} & s_{0} & s_{0} \\
s_{4} & s_{1} & - & s_{4} & s_{1} \\
s_{5} & s_{6} & s_{2} & - & s_{2} \\
s_{6} & s_{6} & s_{5} & s_{4} & -
\end{array}\right) \\
& \bar{L}^{6}=\left(\begin{array}{ccccc}
- & s_{5} & s_{1} & s_{1} & s_{0} \\
s_{1} & - & s_{6} & s_{2} & s_{4} \\
s_{5} & s_{0} & - & s_{3} & s_{1} \\
s_{5} & s_{4} & s_{3} & - & s_{1} \\
s_{6} & s_{2} & s_{5} & s_{5} & -
\end{array}\right)
\end{aligned}
$$




$$
\begin{aligned}
\bar{L}^{7} & =\left(\begin{array}{lllll}
- & s_{0} & s_{1} & s_{1} & s_{1} \\
s_{6} & - & s_{6} & s_{5} & s_{4} \\
S_{5} & s_{0} & - & s_{3} & s_{5} \\
S_{5} & s_{1} & s_{3} & - & s_{2} \\
s_{5} & s_{2} & s_{1} & s_{4} & -
\end{array}\right) \\
\bar{L}^{8} & =\left(\begin{array}{lllll}
- & s_{0} & s_{5} & s_{1} & s_{1} \\
s_{6} & - & s_{6} & s_{4} & s_{5} \\
s_{1} & s_{0} & - & s_{1} & s_{2} \\
s_{5} & s_{2} & s_{5} & - & s_{3} \\
s_{5} & s_{1} & s_{4} & s_{3} & -
\end{array}\right)
\end{aligned}
$$

The $\operatorname{OCI}\left(\bar{L}^{k}\right)$ values $(k=1,2, \ldots, 8)$ are given in Table I.

TABLE I

\begin{tabular}{c|c|c|c|c}
\multicolumn{5}{c}{ OPTIMUM CONSISTENCY OF $\bar{L}^{k}(k=1,2, \ldots, 8)$} \\
\hline & $\bar{L}^{1}$ & $\bar{L}^{2}$ & $\bar{L}^{3}$ & $\bar{L}^{4}$ \\
\hline OCI & 0.833 & 0.7 & 0.948 & 0.783 \\
\hline & $\bar{L}^{5}$ & $\bar{L}^{6}$ & $\bar{L}^{7}$ & $\bar{L}^{8}$ \\
\hline OCI & 0.831 & 0.76 & 0.878 & 0.945 \\
\hline
\end{tabular}

The values of $N S^{k}\left(s_{i}\right)$ for $i=0,1, \ldots, 6 ; k=2,3, \ldots, 8$ are provided in Table II.

TABLE II

PNSS OF LINGUISTIC TERMS FOR DECISION MAKERS $e_{k}(k=2,3, \ldots, 8)$

\begin{tabular}{c|c|c|c|c|c|c|c}
\hline & $N S^{k}\left(s_{0}\right)$ & $N S^{k}\left(s_{1}\right)$ & $N S^{k}\left(s_{2}\right)$ & $N S^{k}\left(s_{3}\right)$ & $N S^{k}\left(s_{4}\right)$ & $N S^{k}\left(s_{5}\right)$ & $N S^{k}\left(s_{6}\right)$ \\
\hline$k=2$ & 0 & 0.25 & - & 0.5 & 0.75 & - & 1 \\
\hline$k=3$ & 0 & 0.245 & 0.255 & 0.5 & 0.745 & 0.76 & 1 \\
\hline$k=4$ & 0 & 0.25 & - & 0.5 & 0.584 & 0.834 & 1 \\
\hline$k=5$ & 0 & 0.244 & 0.2562 & 0.5 & 0.584 & 0.76 & 1 \\
\hline$k=6$ & 0 & 0.25 & 0.271 & 0.5 & 0.584 & 0.76 & 1 \\
\hline$k=7$ & 0 & 0.25 & 0.4 & 0.5 & 0.75 & 0.76 & 1 \\
\hline
\end{tabular}

Fig.3 shows the difference among the PNSs of linguistic terms for $e_{k}(k=1,2, \ldots, 8)$.

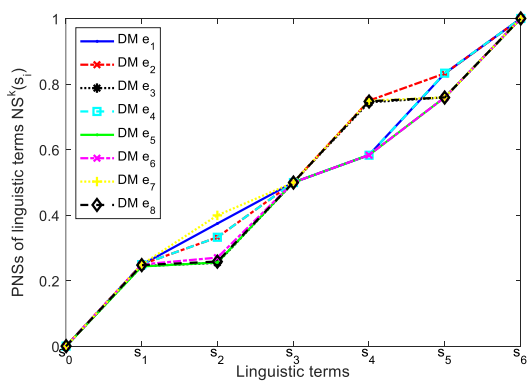

Fig.3. PNSs of linguistic terms for decision makers

From the numerical analysis we can see that the proposed methodology with PISs for managing incomplete linguistic preference relations results in different personalized numerical meanings for different decision makers and guarantees the optimum consistency of linguistic expressions, i.e. it reflects the different understandings of words by different decision makers.

\section{COMPARATIVE STUDY}

In this section, we make a numerical analysis comparison with the existing method to manage incomplete linguistic preference relations that does not consider the decision makers' PISs.

Without personalizing individual semantics, the decision makers are assumed to have the same word semantics. In this case, instead of the numerical scale model, the 2-tuple linguistic model [14] is applied as the linguistic computational model for computing with words. In other words, the numerical scale function $N S^{k}$ is replaced by the function $\Delta^{-1}$ in the computation process, and the semantics of linguistic terms $\left\{s_{0}, s_{1}, \ldots, s_{g}\right\}$ for all the decision makers are $\{0,1, \ldots, g\}$ because $\Delta^{-1}\left(s_{i}\right)=i$.

Let $L^{k}$ be the incomplete linguistic preference relation provided by $e_{k}$, and $V^{k}$ and $A T V^{k}$ as previously defined. Next, we propose an optimization model to estimate missing values of $L^{k}$ based on the 2-tuple linguistic model.

Let $\bar{L}^{k}$ be the complete linguistic preference relation associated to $L^{k}$. Based on Definition 3, we measure the consistency of $\bar{L}^{k}$ as follows,

$$
\begin{gathered}
C I\left(\bar{L}^{k}\right)=1-\frac{2}{3 * A A T V^{k}} \sum_{i<j<z ;(i, j, z) \in A T V^{k}}^{n} \mid \Delta^{-1}\left(\bar{l}_{i j}^{k}\right)+ \\
\Delta^{-1}\left(\bar{l}_{j z}^{k}\right)-\Delta^{-1}\left(\bar{l}_{i z}^{k}\right)-0.5
\end{gathered}
$$

To guarantee the obtained complete linguistic preference relation has maximum consistency index, the objective of the optimization model will be

$$
\max \operatorname{CI}\left(\bar{L}^{k}\right)
$$

In constructing the complete linguistic preference relation $\bar{L}^{k}$, its elements need to fulfill the following conditions,

$$
\begin{cases}l_{i j}^{k}=l_{i j}^{k}, & l_{i j}^{k} \neq \text { null, } \\ l_{i j}^{k} \in S, & l_{i j}^{k}=\text { null }\end{cases}
$$

Therefore, the optimization model to estimate the missing values of $L^{k}$ based on 2-tuple linguistic model is $\left\{\begin{array}{l}\max C I\left(\bar{L}^{k}\right)=1-\frac{4}{n(n-1)(n-2)} \\ \sum_{i<j<z}^{n}\left|\Delta^{-1}\left(\bar{l}_{i j}^{k}\right)+\Delta^{-1}\left(\bar{l}_{j z}^{k}\right)-\Delta^{-1}\left(\bar{l}_{i z}^{k}\right)-0.5\right| \\ \text { s.t. } \\ \bar{l}_{i j}^{k}=l_{i j}^{k}, \quad l_{i j}^{k} \neq \text { null } \\ \bar{l}_{i j}^{k} \in S, \quad l_{i j}^{k}=\text { null }\end{array}\right.$

Solving the above model will result in the complete linguistic preference relations $\bar{L}^{k}(k=1,2, \ldots, m)$ and the corresponding consistency index $C I\left(\bar{L}^{k}\right)$.

To illustrate the above model and to make a comparison with the proposed methodology with PISs, we provide numerical analysis using the set of eight incomplete preference relations $L^{k}(k=1,2 \ldots, 8)$ of Section IV.

Based on the 2-tuple linguistic model, the following semantics of linguistic terms for decision makers are obtained: $\Delta^{-1}\left(s_{0}\right)=0 ; \Delta^{-1}\left(s_{1}\right)=0.167 ; \Delta^{-1}\left(s_{2}\right)=0.333 ; \Delta^{-1}\left(s_{3}\right)=$ $0.5 ; \Delta^{-1}\left(s_{4}\right)=0.667 ; \Delta^{-1}\left(s_{5}\right)=0.833$ and $\Delta^{-1}\left(s_{6}\right)=1$.

The optimization model to estimate the missing values of $L^{1}$ is

$$
\begin{aligned}
& \max C I\left(\bar{L}^{1}\right)=1-\frac{4}{n(n-1)(n-2)} \sum_{i<j<z}^{5} \mid \Delta^{-1}\left(\bar{l}_{i j}^{1}\right)+\Delta^{-1}\left(\bar{l}_{j z}^{1}\right) \\
& -\Delta^{-1}\left(\bar{l}_{i z}^{1}\right)-0.5 \mid
\end{aligned}
$$


subject to

$\bar{l}_{12}^{1}=s_{4} ; \bar{l}_{13}^{1}=s_{1} ; \bar{l}_{23}^{1}=s_{2}$,

$\bar{l}_{24}^{1}=s_{3} ; \bar{l}_{34}^{1}=s_{0} ; \bar{l}_{45}^{1}=s_{2}$,

$\bar{l}_{14}^{1} \in S ; \bar{l}_{15}^{1} \in S ; \bar{l}_{25}^{1} \in S ; \bar{l}_{35}^{1} \in S$.

By solving Eqs. (41)-(44), the following missing value estimations of $L^{1}$ are obtained: $\bar{l}_{14}^{1}=s_{4}, \bar{l}_{15}^{1}=s_{3}, \bar{l}_{25}^{1}=s_{2}$ and $\bar{l}_{35}^{1}=s_{3}$. Thus, the complete linguistic preference relation $\bar{L}^{1}$ is

$$
\bar{L}^{1}=\left(\begin{array}{ccccc}
- & s_{4} & s_{1} & s_{4} & s_{3} \\
s_{2} & - & s_{2} & s_{3} & s_{2} \\
s_{5} & s_{4} & - & s_{0} & s_{3} \\
s_{2} & s_{3} & s_{6} & - & s_{2} \\
s_{3} & s_{4} & s_{3} & s_{4} & -
\end{array}\right)
$$

Similarly, we estimate the missing values of $L^{k}(k=$ $2,3, \ldots, 8)$, which results in the following complete linguistic preference relations $\bar{L}^{k}(k=2,3, \ldots, 8)$ :

$$
\begin{aligned}
& \bar{L}^{2}=\left(\begin{array}{ccccc}
- & s_{6} & s_{0} & s_{0} & s_{0} \\
s_{0} & - & s_{3} & s_{3} & s_{4} \\
s_{6} & s_{3} & - & s_{3} & s_{4} \\
s_{6} & s_{3} & s_{3} & - & s_{6} \\
s_{6} & s_{2} & s_{2} & s_{0} & -
\end{array}\right) \\
& \bar{L}^{3}=\left(\begin{array}{ccccc}
- & s_{5} & s_{3} & s_{6} & s_{2} \\
s_{1} & - & s_{1} & s_{5} & s_{0} \\
s_{3} & s_{5} & - & s_{6} & s_{2} \\
s_{0} & s_{1} & s_{0} & - & s_{0} \\
s_{4} & s_{6} & s_{4} & s_{6} & -
\end{array}\right) \\
& \bar{L}^{4}=\left(\begin{array}{ccccc}
- & s_{1} & s_{0} & s_{4} & s_{0} \\
s_{5} & - & s_{2} & s_{5} & s_{2} \\
s_{6} & s_{4} & - & s_{6} & s_{3} \\
s_{2} & s_{1} & s_{0} & - & s_{6} \\
s_{6} & s_{4} & s_{3} & s_{0} & -
\end{array}\right) \\
& \bar{L}^{5}=\left(\begin{array}{ccccc}
- & s_{0} & s_{2} & s_{1} & s_{0} \\
s_{6} & - & s_{5} & s_{0} & s_{3} \\
s_{4} & s_{1} & - & s_{4} & s_{1} \\
s_{5} & s_{6} & s_{2} & - & s_{2} \\
s_{6} & s_{3} & s_{5} & s_{4} & -
\end{array}\right) \\
& \bar{L}^{6}=\left(\begin{array}{ccccc}
- & s_{5} & s_{1} & s_{1} & s_{0} \\
s_{1} & - & s_{6} & s_{2} & s_{4} \\
s_{5} & s_{0} & - & s_{3} & s_{2} \\
s_{5} & s_{4} & s_{3} & - & s_{2} \\
s_{6} & s_{2} & s_{4} & s_{4} & -
\end{array}\right) \\
& \bar{L}^{7}=\left(\begin{array}{ccccc}
- & s_{0} & s_{3} & s_{2} & s_{1} \\
s_{6} & - & s_{6} & s_{5} & s_{4} \\
s_{3} & s_{0} & - & s_{2} & s_{5} \\
s_{4} & s_{1} & s_{4} & - & s_{2} \\
s_{5} & s_{2} & s_{1} & s_{4} & -
\end{array}\right) \\
& \bar{L}^{8}=\left(\begin{array}{ccccc}
- & s_{0} & s_{5} & s_{1} & s_{1} \\
s_{6} & - & s_{6} & s_{4} & s_{5} \\
s_{1} & s_{0} & - & s_{1} & s_{2} \\
s_{5} & s_{2} & s_{5} & - & s_{3} \\
s_{5} & s_{1} & s_{4} & s_{3} & -
\end{array}\right)
\end{aligned}
$$

The consistency index of the complete linguistic preference relations $\bar{L}^{k}(k=1,2, \ldots, 8)$ are provided in Table III.

TABLE III

CONSISTENCY INDEX OF $\bar{L}^{k}(k=1,2, \ldots, 8)$

\begin{tabular}{c|c|c|c|c}
\multicolumn{5}{|c}{ CONSISTENCY INDEX OF $\bar{L}^{k}(k=1,2, \ldots, 8)$} \\
\hline & $\bar{L}^{1}$ & $\bar{L}^{2}$ & $\bar{L}^{3}$ & $\bar{L}^{4}$ \\
\hline$C I$ & 0.8 & 0.7 & 0.9334 & 0.767 \\
\hline
\end{tabular}

\begin{tabular}{c|c|c|c|c}
\hline & $\bar{L}^{5}$ & $\bar{L}^{6}$ & $\bar{L}^{7}$ & $\bar{L}^{8}$ \\
\hline$C I$ & 0.8 & 0.733 & 0.866 & 0.889 \\
\hline
\end{tabular}

From the comparison of the consistency indexes of Table III with the ones obtained from the proposed PIS method of Table I, it is obvious that the complete linguistic preference relations have lower consistency without considering PISs than considering PISs, i.e. $\operatorname{CI}\left(\bar{L}^{k}\right) \leq O C I\left(\bar{L}^{k}\right)$. It can be observed in the case that PISs are not implemented, the semantics of the linguistic terms are fixed, which results the consistency of the complete linguistic preference relations is fixed as well. While in our proposed method, we obtain the PNSs of linguistic terms to guarantee the optimum consistency of the complete linguistic preference relations, which makes the higher consistency than that in the case without the consideration of PISs. The reason behind these results can be explained: not only the linguistic terms in a preference relation but also their semantics will strongly influence the consistency degree of a linguistic preference relation. In the literature of linguistic consistency, this issue has been ignored.

Therefore, it is shown that our methodology is more effective for managing incomplete linguistic preference relations because not only is able to model the different understandings of words by different decision makers, as expected in real cases, but also achieves completed linguistic preference relations with higher consistency index on the basis of consistency.

\section{CONCLUSION}

Words mean different things for different people, that is, decision makes have PISs regarding words. Incorporating the decision makers' PISs in LGDM results in a novel more realistic and effective methodology for managing incomplete linguistic preference relations. This paper proposes a consistency-driven two-phase methodology based on PISs to manage incomplete linguistic preference relations with two optimization models. Specifically, the first phase PIS-CDO model is developed to obtain optimum consistency of incomplete linguistic preference relation by establishing the sets of possible PNSs, which are subsequently integrated in the second phase IPE-CDO model to estimate the missing values of the incomplete linguistic preference relation based on PISs and the personalized numerical meanings for linguistic terms of the different decision makers.

The personalization of linguistic expressions and the estimation of missing values based on consistency measurement in the process of managing incomplete linguistic preference relations are evidence of the advantages of our proposed methodology. In this paper, the LGDM is based on the use of well-established but simple linguistic terms set and additive consistency. In future, we will investigate methods to manage incomplete linguistic information with ordinal consistency [43] and complex linguistic expressions, such as hesitant linguistic term sets [29], [33], [36], [39], linguistic distribution [38], [47], [50], flexible linguistic expression [37] and heterogeneous information [16]. 


\section{References}

[1] S. Alonso, F. Chiclana, F. Herrera, E. Herrera-Viedma, J. Alcalá-Fdez, and C. Porcel, "A consistency-based procedure to estimate missing pairwise preference values," Int. J. Intell. Syst., vol. 23, no. 2, pp. 155-175, 2008.

[2] G. Bordogna, M. Fedrizzi, and G. Pasi, "A linguistic modeling of consensus in group decision making based on OWA operators," IEEE Trans. Syst. Man Cybern. Part A Syst. Humans, vol. 27, no. 1, pp. 126-133, 1997.

[3] F.J. Cabrerizo, I.J. Pérez, and E. Herrera-Viedma, "Managing the consensus in group decision making in an unbalanced fuzzy linguistic context with incomplete information," Knowl-Based Syst., vol. 23, no. 2, pp. 169-181, 2010.

[4] M. Cai, Z.W. Gong, and J. Cao, "The consistency measures of multi-granularity linguistic group decision making," J. Intell. Fuzzy Syst., vol. 29, no. 2, pp. 609-618, 2015.

[5] K. Chen, G. Kou, J.M. Tarn, and Y. Song, "Bridging the gap between missing and inconsistent values in eliciting preference from pairwise comparison matrices," Ann. Oper. Res., vol. 235, pp. 155-175, 2015.

[6] Y.C. Dong, Y. Xu, and S. Yu, "Computing the numerical scale of the linguistic term set for the 2-tuple fuzzy linguistic representation model," IEEE Trans. Fuzzy Syst., vol. 17, no. 6, pp. 1366-1378, 2009.

[7] Y.C. Dong, C.C. Li, and F. Herrera, "Connecting the linguistic hierarchy and the numerical scale for the 2-tuple linguistic model and its uses to deal with hesitant unbalanced linguistic information," Inf. Sci., vol. 367-368, pp. 259-278, 2016.

[8] Y.C. Dong and E. Herrera-Viedma, "Consistency-driven automatic methodology to set interval numerical scales of 2-tuple linguistic term sets and its use in the linguistic GDM with preference relation," IEEE Trans. Cybern., vol. 45, no. 4, pp. 780-792, 2015.

[9] M. Fedrizzi, and S. Giove, "Incomplete pairwise comparison and consistency optimization,” Eur. J. Oper. Res., vol. 183, pp. 303-313, 2007.

[10] Z.W. Gong, C. Guo, and Y. He, "Group decision making methods of the incomplete IFPRs and IPRs," Int. J. of Comput. Intell. Syst., vol. 5, no. 3, pp. 542-552, 2012.

[11] F. Herrera, E. Herrera-Viedma, and J.L. Verdegay, "A linguistic decision process in group decision making," Group Decis. Negot., vol. 5, no. 2, pp. 165-176, 1996.

[12] F. Herrera, E. Herrera-Viedma, and J.L. Verdegay, "Preference degrees over linguistic preference relations in decision making," Oper. Res. Decis., vol. 3, pp. 37-48, 1995.

[13] F. Herrera, E. Herrera-Viedma, and J.L. Verdegay, "A sequential selection process in group decision making with a linguistic assessment approach," Inf. Sci., vol. 85, pp. 223-239, 1995.

[14] F. Herrera and L. Martínez, "A 2-tuple fuzzy linguistic representation model for computing with words," IEEE Trans. Fuzzy Syst., vol. 8, no. 6, pp. 746-752, 2000

[15] E. Herrera-Viedma, F. Chiclana, F. Herrera, and S. Alonso, "Group decision-making model with incomplete fuzzy preference relations based on additive consistency," IEEE Trans. Syst. Man Cybern. Part B Cybern., vol. 37, no. 1, pp. 176-189, 2007.

[16] E. Herrera-Viedma, F. Herrera, and F. Chiclana, "A consensus model for multiperson decision making with different preference structures," IEEE Trans. Syst. Man Cybern. Part A Syst. Humans, vol. 32, no. 3, pp, 394-402, 2002.

[17] H. Huang, C. Li, and S. Member, "Extended personalized individual semantics with 2-tuple linguistic preference for supporting consensus decision making," IEICE Trans. Inf. Syst., vol. 101, pp. 387-395, 2018.

[18] C.C. Li, Y.C. Dong, F. Herrera, E. Herrera-Viedma, and L. Martínez, "Personalized individual semantics in computing with words for supporting linguistic group decision making. An application on consensus reaching," Inf. Fusion, vol. 33, pp. 29-40, 2017.

[19] C.C. Li, R.M. Rodríguez, L. Martínez, Y.C. Dong, and F. Herrera, "Personalized individual semantics based on consistency in hesitant linguistic group decision making with comparative linguistic expressions,” Knowl-Based Syst., vol. 145, pp. 156-165, 2018.

[20] C.C. Li, Y.C. Dong, and F. Herrera, "A consensus model for large-scale linguistic group decision making with a feedback recommendation based on clustered personalized individual semantics and opposing consensus groups," IEEE Trans. Fuzzy Syst., vol. 27, no. 2, pp. 221-233, 2019.

[21] C.C. Li, Y.C. Dong, Y.X. Xu, F. Chiclana, E. Herrera-Viedma, and F. Herrera, "An overview on managing additive consistency of reciprocal preference relations for consistency-driven decision making and fusion: Taxonomy and future directions," Inf. Fusion, vol. 52, pp. 143-156, 2019.
[22] F. Liu, W.G. Zhang, "Topsis-based consensus model for group decision-making with incomplete interval fuzzy preference relations," IEEE Trans. Cybern., vol. 44, no. 8, pp. 1283-1294, 2013.

[23] H. Liang, C.C. Li, Y.C. Dong and F. Herrera, "Linguistic Opinions Dynamics based on Personalized Individual Semantics," IEEE Trans. Fuzzy Syst., doi: 10.1109/TFUZZ.2020.2999742.

[24] L. Martínez, and F. Herrera, "An overview on the 2-tuple linguistic model for computing with words in decision making: Extensions, applications and challenges," Inf. Sci., vol. 207, pp. 1-18, 2012.

[25] J.M. Mendel and D. Wu, Perceptual Computing: Aiding People in Making Subjective Judgments. New York, NY, USA: IEEE-Wiley, 2010.

[26] J.M. Mendel, L.A. Zadeh, E. Trillas, R.R. Yager, J. Lawry, H. Hagras, and S. Guadarrama, "What computing with words means to me: Discussion forum," IEEE Comput. Intell. M., vol. 5, no. 1, pp. 20-26, 2010.

[27] F. Meng and X. Chen, "A new method for group decision making with incomplete fuzzy preference relations," Knowl. Based Syst., vol. 73, pp. 111-123, 2015.

[28] F. Meng and X. Chen, "An approach to incomplete multiplicative preference relations and its application in group decision making," Inf. Sci., vol. 309 pp. 119-137, 2015.

[29] R.M. Rodríguez, L. Martínez, and F. Herrera, "Hesitant fuzzy linguistic term sets for decision making," IEEE Trans. Fuzzy Syst., vol. 20, no. 1, pp. 109-119, 2012.

[30] X. Tang, Q. Zhang, Z. Peng, S. Yang, and W. Pedrycz, "Derivation of personalized numerical scales from distribution linguistic preference relations: an expected consistency-based goal programming approach," Neural Comput. Appl., vol. 31, no. 12, pp. 8769-8786, 2019.

[31] X. Tang, Q. Zhang, Z. Peng, W. Pedrycz, and S. Yang, "Distribution linguistic preference relations with incomplete symbolic proportions for group decision making," Appl. Soft Comput., vol. 88, 106005, 2020.

[32] R. Ureña, F. Chiclana, J.A. Morente-Molinera, and E. Herrera-Viedma, "Managing incomplete preference relations in decision making: a review and future trends," Inf. Sci., vol. 302, no. 1, pp. 14-32, 2015.

[33] L.H. Wang and Z.W. Gong, "Priority of a hesitant fuzzy linguistic preference relation with a normal distribution in meteorological disaster risk assessment," Int. J. Environ. Res. Pub. Health, vol. 14, no. 10, 1203, 2017.

[34] Z.J. Wang, A note on "incomplete interval fuzzy preference relations and their applications ," Comput. Ind. Eng., vol. 77, pp. 65-69, 2014.

[35] Z.J. Wang, A note on "A goal programming model for incomplete interval multiplicative preference relations and its application in group decision-making, " Eur. J. Oper. Res., vol. 247, pp. 867-871, 2015.

[36] C.P. Wei, R.M. Rodríguez, and L. Martínez, "Uncertainty measures of extended hesitant fuzzy linguistic term sets," IEEE Trans. Fuzzy Syst., vol. 26, no. 3, pp. 1763-1768, 2018

[37] Y.Z. Wu, Y.C. Dong, J. Qin, and W. Pedrycz, "Flexible linguistic expressions and consensus reaching with accurate constraints in group decision-making," IEEE Trans. Cybern., vol. 50, no. 6, pp. 2488-2501, 2020.

[38] Y.Z. Wu, C.C. Li, X. Chen, and Y.C. Dong, "Group decision making based on linguistic distributions and hesitant assessments: Maximizing the support degree with an accuracy constraint," Inf. Fusion, vol. 41, pp. 151-160, 2018.

[39] Z. B. Wu and J. P. Xu, "Possibility distribution-based approach for MAGDM with hesitant fuzzy linguistic information," IEEE Trans. Cybern., vol. 46, no. 3, pp. 694-705, 2016.

[40] J.P. Xu and Z.B. Wu, "A maximizing consensus approach for alternative selection based on uncertain linguistic preference relations," Comput. Ind. Eng., vol. 64, no. 4, pp. 999-1008, 2013.

[41] Y.J. $\mathrm{Xu}$ and F. Herrera, "Visualizing and rectifying different inconsistencies for fuzzy reciprocal preference relations," Fuzzy Sets Syst., vol. 362, pp. 85-109, 2019.

[42] Y.J. Xu, C.P. Wei, and H. Sun, "Distance-based nonlinear programming models to identify and adjust inconsistencies for linguistic preference relations," Soft Comput., vol. 22, no. 14, pp. 4833-4849, 2018.

[43] Y.J. Xu, F. Ma, and F. Herrera, "Revisiting inconsistent judgments for incomplete fuzzy linguistic preference relations: Algorithms to identify and rectify ordinal inconsistencies," Knowl-Based Syst., vol. 163, pp. 305-319, 2019.

[44] Y.J. Xu , K.W. Li , and H.M. Wang, "Incomplete interval fuzzy preference relations and their applications," Comput. Ind. Eng., vol. 67, pp. 93-103, 2014.

[45] M. Zhao, X.Y. Ma, and D.W. Wei, "A method considering and adjusting individual consistency and group consensus for group decision making 
with incomplete linguistic preference relations," Appl. Soft Comput., vol. 54, pp. 322-346, 2017.

[46] R. Yager, "An approach to ordinal decision making," Int. J. Approx. Reason., vol. 12, pp. 237-261, 1995.

[47] Z. Zhang, C. Guo, and L. Martínez, "Managing multigranular linguistic distribution assessments in large-scale multiattribute group decision making," IEEE Trans. Syst. Man Cybern. Syst., vol. 47, no. 11, pp. 3063-3076, 2017.

[48] H. Zhang, C.C. Li, Y. Liu and Y.C. Dong, "Modelling personalized individual semantics and consensus in comparative linguistic expression preference relations with self-confidence: An optimization-based approach," IEEE Trans. Fuzzy Syst., doi: 10.1109/TFUZZ.2019.2957259.

[49] H. Zhang, Y.C. Dong, J. Xiao, F. Chiclana and E. Herrera-Viedma, "Personalized individual semantics-based approach for linguistic failure modes and effects analysis with incomplete preference information," IISE Trans., vol. 52, no. 11, pp. 1275-1296, 2020.

[50] G.Q. Zhang, Y.C. Dong, and Y.F. Xu, "Consistency and consensus measures for linguistic preference relations based on distribution assessments," Inf. Fusion, vol. 17, pp. 46-55, 2014.

[51] G.Q. Zhang, Y.C. Dong, and Y.F. Xu, "Linear optimization modeling of consistency issues in group decision making based on fuzzy preference relations," Expert Syst. Appl., vol. 39, no. 3, pp. 2415-2420, 2012.

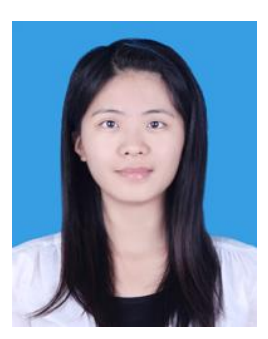

Cong-Cong $\mathbf{L i}$ received the Ph.D. degree in management from Sichuan University, Chengdu, China, in 2018, and the Ph.D. degree in computer science and artificial intelligence from the University of Granada, Granada, Spain, both in 2018. She is currently a Lecturer with the School of Economics and Management, Southwest Jiaotong University, Chengdu.

Her research interests include decision making, computing with words, and opinion dynamics. Her research results have been published in some refereed journals, including IEEE TRANSACTIONS ON CYBERNETIC, IEEE TRANSACTIONS ON FUZZY SYSTEMS, IEEE TRANSACTIONS ON SYSTEMS, MAN, AND CYBERNETICS: SYSTEMS, Information Fusion, Information Sciences, and Group Decision and Negotiation.

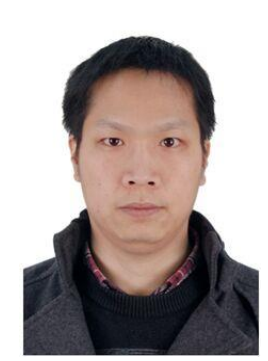

Yucheng Dong (Senior Member, IEEE) received the B.S. and M.S. degrees in mathematics from Chongqing University, Chongqing, China, in 2002 and 2004, respectively, and the Ph.D. degree in management from Xi'an Jiaotong University, Xi'an, China, in 2008.

$\mathrm{He}$ is currently a Professor with the Business School, Sichuan University, Chengdu, China. He has authored or coauthored more than 100 international journal papers in in some refereed journals. His current research interests include decision analysis, human dynamics, social network, and data science. He has been identified by Clarivate as Highly Cited Researcher in the field of computer science.

Prof. Dong is an Area Editor/Associate Editor of Computers and Industrial Engineering, Group Decision and Negotiation, the IEEE TRANSACTIONS ON SYSTEMS, MAN, AND CYBERNETICS: SYSTEMS, and Information Fusion.

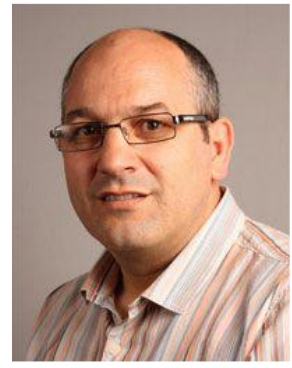

Francisco Chiclana received the B.Sc. and Ph.D. degrees in mathematics from the University of Granada, Granada, Spain, in 1989 and 2000, respectively.

He is a Professor with the Institute of Artificial Intelligence and School of Computer Science and Informatics, De Montfort University, Leicester, U.K.

Prof. Chiclana is currently a Highly Cited Researcher in Computer Sciences (according to Essential Science Indicators by Clarivate Analytics). He is an Associate Editor and a Guest Editor for several ISI indexed journals. He has organized and chaired special sessions/workshops in many major international conferences in research areas as fuzzy preference modeling, decision support systems, consensus, recommender systems, social networks, rationality/consistency, and aggregation.

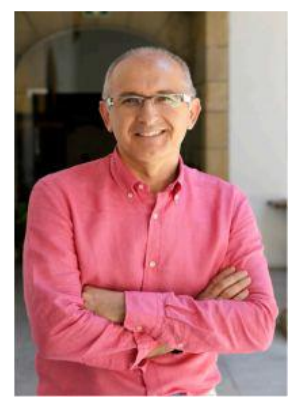

Enrique Herrera-Viedma (Fellow Member, IEEE) received the M.Sc. and Ph.D. degrees in computer science from the University of Granada, Granada, Spain, in 1993 and 1996, respectively.

$\mathrm{He}$ is a Professor of computer science and the Vice-President for Research and Knowledge Transfer with University of Granada, Granada, Spain. His h-index is 82 with more than 24000 citations received in Web of Science and 100 in Google Scholar with more than 40000 cites received. He has been identified as one of the world's most influential researchers by the Shanghai Center and Thomson Reuters/Clarivate Analytics in both computer science and engineering in the years 2014, 2015, 2016, 2017, 2018, 2019 and 2020. His current research interests include group decision making, consensus models, linguistic modeling, aggregation of information, information retrieval, bibliometric, digital libraries, web quality evaluation, recommender systems, and social media.

Dr. Herrera-Viedma is IEEE Fellow and Vice-President for Cybernetics in IEEE SMC and an Associate Editor in several journals such as IEEE Transactions on Fuzzy Systems, IEEE Transactions on Systems, Man, and Cybernetics: Systems, IEEE Transactions on Intelligent Transport System, Information Sciences, Applied Soft Computing, Soft Computing, Fuzzy Optimization and Decision Making, and Knowledge-Based Systems. 